\title{
Reduced Calorie Sweetener Use Does Not Adversely Affect Gastrointestinal Health and Function
}

TO THE EDITOR: Spencer et al ${ }^{1}$ aimed to identify the effects of artificial sweeteners (AS) on the gastrointestinal (GI) tract "to determine whether they could be playing a role in GI symptoms in persons with irritable bowel syndrome (IBS)". They found "no studies on the effects of AS on GI symptoms in the general population, or more specifically in IBS patients".

Spencer et $\mathrm{al}^{1}$ hypothesized that AS have the potential to affect the GI system since AS can interact with sweet taste receptors in the GI tract. While AS, like nutritive sugars, interact with gut sweet taste receptors, in vivo research collectively supports that this interaction does not lead to clinically relevant changes in GI health. In a recent review, Bryant and Mclaughlin ${ }^{2}$ reported that human gut exposure to AS consistently "fails to replicate any of the effects on gastric motility, gut hormones or appetitive responses evoked by caloric sugars." Likewise, the majority of in vivo laboratory animal research shows no clinically meaningful changes in GI hormones associated with taste receptor activation by $\mathrm{AS}^{2-4}$

Robust research demonstrates the safety of AS in both humans and animal models and led to approval of these sweeteners as food additives by regulatory agencies. It should be noted that many studies reviewed by regulatory agencies around the world have particu- larly assessed the potential for effects on GI heath and found them to have no effect. This is based on numerous types of measures, including, for example, evaluation of hematologic and biochemical analyses, macroscopic and microscopic examination of GI tissues.

The collective literature clearly supports that human consumption of AS is without adverse effect on GI health and function.

Sylvia P Poulos

Calorie Control Council, Atlanta, GA, USA

1. Spencer M, Gupta A, Dam LV, Shannon C, Menees S, Chey WD. Artificial sweeteners: a systematic review and primer for gastroenterologists. J Neurogastroenterol Motil 2016;22:168-180.

2. Bryant C, Mclaughlin J. Low calorie sweeteners: evidence remains lacking for effects on human gut function. Physiol Behav 2016;164(Pt B):482-485.

3. Renwick AG, Molinary SV. Sweet-taste receptors, low-energy sweeteners, glucose absorption and insulin release. Br J Nutr 2010;104:1415-1420.

4. Brown RJ, Rother KI. Non-nutritive sweeteners and their role in the gastrointestinal tract. J Clin Endocrinol Metab 2012;2597-2605.

Conflicts of interest: None. 